\title{
Experiencia "tricantina" de evaluación formativa y compartida en Educación Primaria
}

\section{"Tricantina" experience of formative and shared evaluation in Primary Education}

\author{
Vanesa Reyes Alonso
}

\author{
Fecha de recepción: 23/01/2019; Fecha de revisión: 25/02/2019; Fecha de aceptación: 25/03/2019
}

Cómo citar este artículo:

Reyes, V. (2019). Experiencia "tricantina" de evaluación formativa y compartida en Educación Primaria. Revista de Innovación y Buenas Prácticas Docentes, 8, 132-141.

Autor de Correspondencia: vanesa_91_sg@hotmail.com

\begin{abstract}
Resumen:
En este trabajo, el cual está vinculado al Proyecto de Innovación Docente titulado "La evaluación formativa y compartida en la educación. Transferencia de conocimiento entre Universidad y Escuela", se explica cómo se ha llevado a cabo la evaluación compartida y formativa a lo largo del curso académico 2017-2018 en un colegio de Tres Cantos (Madrid) con 38 alumnos de $4^{\circ}$ de Educación Primaria en la asignatura de Educación Física. En él, encontramos las diferentes técnicas e instrumentos utilizados, los cuales ayudan a que los alumnos sean partícipes tanto de su proceso de aprendizaje como de su propio proceso de evaluación. Entre ellos, cabe resaltar la autoevaluación y la coevaluación, todo ello ambientado a través de un hilo conductor que lleva como protagonistas a los Pokemons, intentando así que sea más motivante para ellos. En cuanto a los resultados obtenidos, encontramos una notable mejora según van avanzando los trimestres y van siendo conscientes de esta forma de evaluación, ya que como vemos, importa más el proceso que el resultado. Además, podemos observar que es algo a lo que nos están acostumbrados, pero que con el tiempo permite obtener grandes ventajas, mejorando principalmente el aprendizaje del alumnado y su capacidad reflexiva.
\end{abstract}

Palabras clave: Evaluación, Compartida, Formativa, Educación Física.

Abstract:

This report, which is linked to the Teaching Innovation Project entitled "Formative and shared evaluation in education. Transfer of knowledge between University and School ", explains how shared and formative assessments for the subject of Physical Education were carried out on 38 year 4 primary students during the academic year 2017/2018 in the Tres Cantos school in Madrid. It covers the different techniques and tools that were used, and which help students to participate in the learning process as well as in their own assessment. Amongst these, self-assessment and peer assessment stand out. All of it has been done through the Pokemon characters in order to be more motivating for the students. With respect to the results that were achieved, we have found a significant improvement as the terms progressed, and the students became more aware of this type of assessment, because as we can see, the process is more important than the result. We can also see that it is something that we are getting used to, and with time will help to improve the learning ability and reflexive capacity of the students which is a great advantage.

Key Words: Assessment/Evaluation, Shared, Formative, Physical Education. 


\section{INTRODUCCIÓN}

La evolución de las diferentes leyes educativas (LGE, LODE, LOGSE, LOCE, LOE y la actual LOMCE) han provocado, junto a los avances de la tecnología, de la sociedad, etc., el desarrollo de nuevas estrategias de enseñanza y aprendizaje, haciendo que la evaluación en el ámbito educativo haya ido cambiando.

Así, vemos que la evaluación es considerada, un elemento clave en la práctica educativa. Aunque antiguamente, la evaluación se reflejaba en una mera calificación, en la actualidad, Ruíz Nebrera (2008) expone que el maestro no debe evaluar solo para calificar, sino para mejorar los procesos de enseñanza y aprendizaje.

Por eso y con la mirada puesta en cambiar el sentido de la evaluación, hemos desarrollado una propuesta de evaluación formativa y compartida en la asiganatura de Educación Física dentro de la Educación Primaria, encaminada, a mejorar los procesos de enseñanza y aprendizaje de cara a lograr aprendizajes significativos y convertir a nuestros alumnos en protagonistas de la evaluación.

\subsection{Contexto}

El centro con el que se ha aplicado este sistema de evaluación es el CEIP Miguel de Cervantes, que educa alumnos de Educación Infantil y Primaria y es de línea dos. Este centro está ubicado en la localidad de Tres Cantos, en la zona norte de la Comunidad de Madrid.

Respecto a las características del centro, cuenta con un edificio para primaria (de dos plantas) y otro para infantil, además de dos patios (equipados con canastas), una pista de fútbol y un pabellón polideportivo anexo, que dispone de material muy variado y que utilizo para realizar las clases de Educación Física.

El ambiente socioeconómico de este centro es de un nivel medio-alto. La mayoría de los familiares son personas activas, cuyos trabajos están relacionados con la administración, la educación, funcionarios, autónomos, hosteleros y constructores.

El curso con el que se ha llevado a cabo este proyecto es $4^{\circ}$ de EP, tanto con el grupo A como con el B en el área de Educación Física. En $4^{\circ} \mathrm{A}$ hay 19 niños y en $4^{\circ}$ $B, 19$. Ambos grupos tienen dos sesiones semanales de 45 minutos, es decir, 1,5 horas a la semana, dado que el centro es bilingüe.

Entre ambos grupos existen notables diferencias, tanto de rendimiento como de actitud. Pero por lo general, son grupos con un nivel intelectual medio-alto, trabajadores y participativos, aunque a veces surgen conflictos. Además, son niños que por lo general aceptan bien a los demás; sin embargo, les cuesta trabajar en grupos mixtos. En general, todos tienen una actitud positiva hacia las clases de EF. En uno de los grupos hay un alumno TEA (Trastorno del Espectro Autista).

\section{DESARROLLO DE LA EXPERIENCIA DE INNOVACIÓN}

\section{- Objetivos del área de EF.}

Lo primero que nos planteamos antes de iniciar este proceso, son una serie de objetivos generales que quiero conseguir en el área de Educación Física. Estos son los siguientes:

- Conocer su cuerpo y la actividad física como medio de disfrute y relación con los demás, así como tomar conciencia de las posibilidades de expresión y comunicación del cuerpo a través de la expresión corporal. 
- Utilizar la cooperación y el diálogo para resolver problemas y conflictos en diferentes actividades y mejorar las relaciones sociales con los compañeros.

- Realizar actividades que ayuden a tomar conciencia de la importancia del ejercicio físico, la higiene, la seguridad y prevención de riesgos, los primeros auxilios, la alimentación y los hábitos posturales sobre la salud.

- Conocer y practicar diferentes deportes a través de los juegos modificados y deportes alternativos, así como realizar juegos del mundo, juegos alternativos, juegos cooperativos y juegos populares y tradicionales.

- Practicar actividades físicas en el medio natural y utilizar las habilidades motrices básicas para adaptar el movimiento a los diferentes juegos y entornos.

\section{- Actividades de aprendizaje.}

Seguidamente, hablamos sobre las actividades de aprendizaje que se dan en mis clases de Educación Física de manera constante.

- Explicaciones iniciales, realizadas al inicio de cada sesión para comentar lo que se va a trabajar, así como los objetivos de la sesión.

- Circuitos, actividades físicas organizadas y juegos motores, realizados a lo largo de las sesiones y enfocados a los contenidos y objetivos que se pretender lograr.

- Proyectos grupales con coevaluación, los cuales se realizan en algunas unidades didácticas.

- Asambleas finales grupales con asignación de puntos (Pokemons), donde reflexionamos en grupo sobre comportamiento y la actitud, el respeto a las normas, materiales y compañeros, la resolución de conflictos y la participación. En base a esto, los alumnos se asignan un pokemon, asociado a una puntuación.

- Cuaderno del alumno (a partir del $2^{\circ}$ trimestre), donde reflexionan sobre la UD, rellenando al final de la misma una serie de apartados: qué palabras han aprendido, qué contenidos han aprendido, cómo se han sentido y qué actividad les ha gustado más, lo que me da información del aprendizaje realizado, así como de las actividades más motivantes. Este cuaderno se recoge al final de cada trimestre (Ver figura 1 ). 


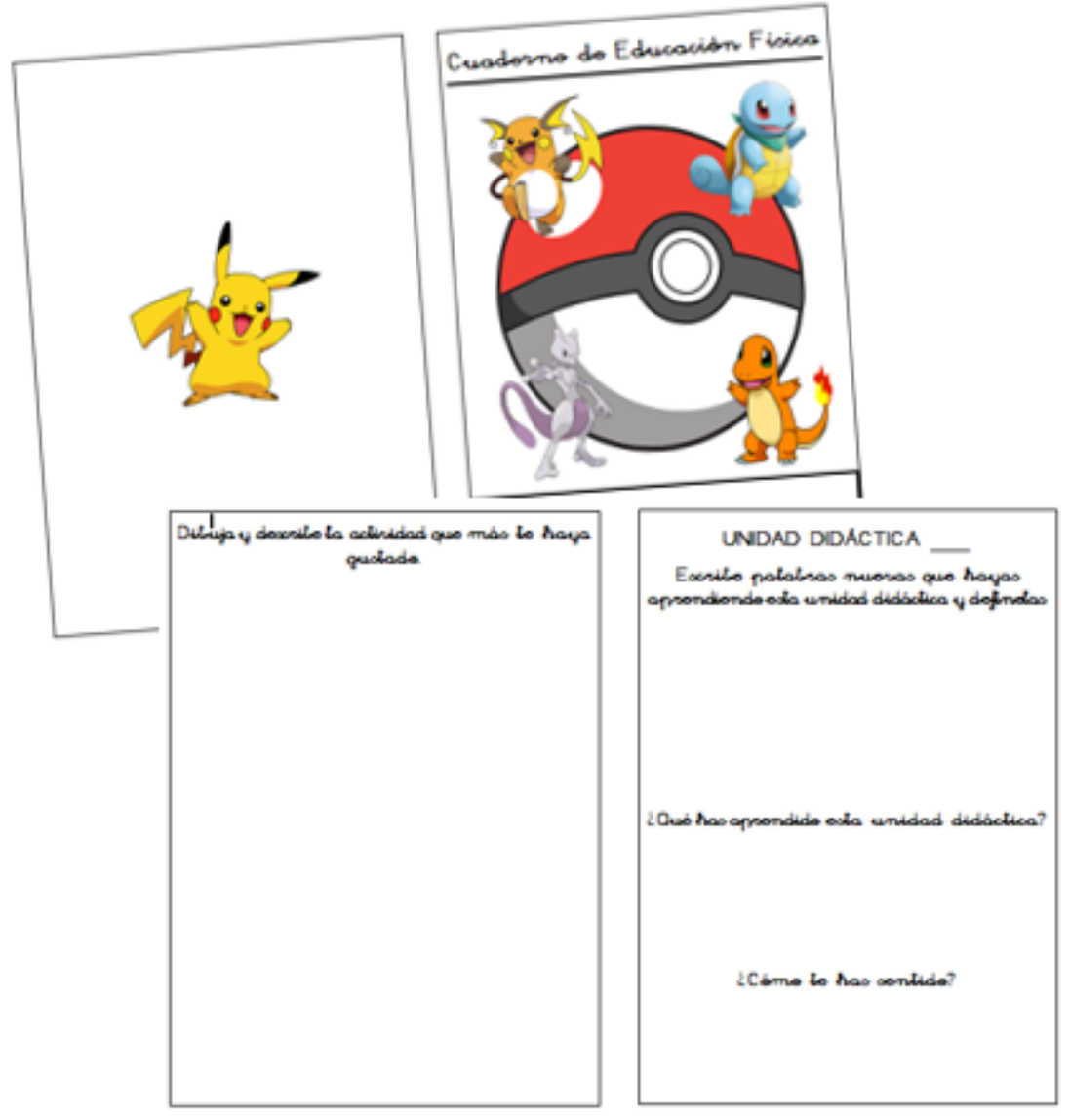

Figura 1. Cuaderno del alumnado. Fuente: Elaboración propia.

\section{- Técnicas e instrumentos.}

A continuación, y basándonos en López-Pastor \& Pérez-Pueyo (2017), vamos a comentar las técnicas e instrumentos que se han utilizado para desarrollar el sistema de evaluación compartida y formativa, teniendo en cuenta el contexto anteriormente comentado. En primer lugar, aparece un cuadro resumen, y a continuación se detalla cada uno de ellos.

\begin{tabular}{|c|c|}
\hline $\begin{array}{l}\text { Técnicas e instrumentos de } \\
\text { evaluación }\end{array}$ & Descripción \\
\hline $\begin{array}{c}\text { Evaluación compartida y } \\
\text { autoevaluación del alumnado }\end{array}$ & $\begin{array}{l}\text { - } \quad \text { Ficha mediante una escala gráfica (pokemons) y } \\
\text { una escala verbal (nada...mucho). }\end{array}$ \\
\hline Coevaluación grupal & $\begin{array}{ll}\text { - } & \text { Ficha entre los miembros de un mismo grupo con } \\
\text { escala verbal. } \\
\text { - } \quad \text { Ficha entre grupos con escala gráfica. }\end{array}$ \\
\hline $\begin{array}{l}\text { Autoevaluación grupal a través } \\
\text { de la asignación de puntos }\end{array}$ & $\begin{array}{l}\text { - Con escala gráfica en la cual cada pokemon lleva } \\
\text { asociado una puntuación. }\end{array}$ \\
\hline
\end{tabular}

Figura 2. Técnicas e instrumentos de evaluación formativa y compartida. 


\begin{tabular}{cll}
\hline Aplicación educativa "Classdojo" & $\begin{array}{l}\text { Puntos extras que complementan la información } \\
\text { obtenida en el diario del maestro. } \\
\text { Se recoger a través de la aplicación y la } \\
\text { información se vuelca en la ficha de calificación. }\end{array}$ \\
\hline $\begin{array}{c}\text { Autoevaluación del maestro y la } \\
\text { unidad didáctica }\end{array}$ & $\begin{array}{l}\text { Ficha con escala verbal que ayuda a mejorar de } \\
\text { cara a futuras unidades didácticas. }\end{array}$ \\
\hline $\begin{array}{c}\text { Ficha de observación grupal con } \\
\text { escala verbal }\end{array}$ & $\begin{array}{l}\text { Ficha con escala verbal, que ofrece la nota de la } \\
\text { unidad didáctica. }\end{array}$ \\
\hline Rúbrica para autoevaluación final & - & $\begin{array}{l}\text { Ficha con escala gráfica (pokemons), escala } \\
\text { verbal y escala numérica. }\end{array}$ \\
\hline Entrevista individual & $-\begin{array}{l}\text { Sobre su rúbrica de autoevaluación se les marca } \\
\text { lo que considero y después realizamos una } \\
\text { entrevista individual para ver si se llega a un } \\
\text { acuerdo. }\end{array}$ \\
\hline \multirow{2}{*}{ Cuaderno del alumno } & $\begin{array}{l}\text { Se recoge al final del trimestre y es obligatorio } \\
\text { para aprobar. Su nota se refleja en la ficha de } \\
\text { calificación. }\end{array}$ \\
\hline
\end{tabular}

Figura 2. Técnicas e instrumentos de evaluación formativa y compartida.

- Evaluación compartida y autoevaluación del alumnado mediante una ficha que rellenan de manera individual al final de cada unidad, la cual es revisada por el maestro, aportando su opinión y después se dialoga sobre ella, realizando una pequeña entrevista individual. En ella, aparecen aspectos sobre los contenidos trabajados en la unidad, el respeto a los compañeros, materiales y normas, la ayuda con el material, etc. Además, en cada ficha de cada unidad se pregunta algún aspecto concreto de lo trabajado en la misma.

- Coevaluación grupal en algunas de las actividades o proyectos realizados a lo largo del curso. Esta puede ser entre los miembros de un grupo, donde cada uno evalúa el trabajo de los compañeros dentro del mismo grupo o entre grupos, donde unos grupos coevaluan el trabajo de otros.

- Autoevaluación grupal a través de la asignación de puntos (Pokemons) en las asambleas finales de cada sesión. En ella, los alumnos establecen un pequeño debate acerca de cómo ha sido su actitud y participación en clase, así como de las cosas que han hecho bien y mal y el respeto a normas y compañeros y se asignan un pokemon, el cual está asociado a una puntuación. Estos pokemons los van registrando en una tabla que tienen en clase y cuando consiguen 50 puntos, se los cambio por una pokeball y obtienen una clase de deporte libre.

- Aplicación educativa "Classdojo", una aplicación que permite otorgar un feedback inmediato a los alumnos desde el móvil, a través de unos ítems positivos y negativos que hacen referencia por ejemplo a la capacidad de reflexión, a la cooperación, el no respeto del material, a la participación, etc. Esta aplicación también ofrece la posibilidad de compartir fotos con ellos. Cada alumno se presenta mediante un avatar (muñeco personalizado), que puede ser modificado por ellos.

Además, admite interactuar con los padres, ya que la aplicación funciona como una plataforma que permite a los padres observar el progreso de sus hijos y mandarme mensajes. Con ella, se contribuye a su vez al fomento de las TIC. Este instrumento complementa la información obtenida del diario del maestro.

- Autoevaluación del maestro y la unidad didáctica a través de una ficha estructurada, que permite comprobar los puntos fuertes y débiles de la unidad didáctica y mejorar las clases de EF. Así, al finalizar cada una de las UUDD, se rellena esta ficha con el objetivo de mejorar de cara a futuras unidades.

- Diario del maestro poco estructurado, tipo anecdotario, formado por un conjunto de folios en blanco, donde se anotan los hechos significativos que van ocurriendo. Además, las anotaciones realizadas giran en torno a la Participación_en clase (intervenciones, predisposición individual para participar y hacer aportaciones, motivación por las actividades, mantenimiento de la atención y actitud positiva); el Trabajo en grupo_(implicación e interés, aportación de ideas relevantes, creación de 
buen ambiente de trabajo); la Capacidad de reflexión_sobre el aprendizaje (valoración de la capacidad individual y de reflexión del aprendizaje durante las sesiones y en las autoevaluaciones); y Otros aspectos (trae chándal, respeto a las normas, a los compañeros y a los materiales).

- Ficha de observación grupal con escala verbal (una para cada unidad didáctica), en la que se evalúa el grado de adquisición de los objetivos a través de una ficha compuesta por una serie de indicadores de logro y una escala verbal, donde quedará reflejado la relación entre los contenidos, los criterios de evaluación y los estándares de aprendizaje evaluables, que van cambiando en cada unidad. Cada uno de estos términos supone un grado distinto de adquisición del indicador de logro, que queda definido en la ficha. Esta ficha de heteroevaluación, también posibilita valorar a qué competencias se está contribuyendo.

- Rúbrica para autoevaluación y autocalificación final del alumnado con escala gráfica (pokemon) y escala verbal (mucho... nada). Esta ficha es completada por los alumnos de manera individual al finalizar cada uno de los trimestres. En ella se recogen diferentes aspectos ya trabajados a lo largo del trimestre y además, permite comparar lo que los alumnos consideran que merecen y lo que el maestro les pone en cada uno de los apartados, viendo así si son conscientes de su trabajo realizado. En el tercer trimestre y dentro de esta misma ficha, se añade la autocalificación.

- Escala graduada. A principios del segundo trimestre se elabora una escala graduada, donde aparece qué deben hacer los alumnos para sacar $X$ nota en la asignatura de EF.

En cuanto al uso del feedback, se utiliza el intrínseco y extrínseco, intentando así, que el aprendizaje significativo. Este feedback se da a lo largo de las sesiones de manera individual y grupal, tanto durante la propia actividad o juego motriz como al final de la misma, dependiendo de su dificultad y de si queremos centrarnos más en la ejecución o en los resultados.

Además, realizamos diferentes asambleas, donde tanto el maestro como los propios alumnos dan feedback a sus compañeros. Y por último, se otorga feedback a los alumnos tanto en las fichas de autoevaluación, como de coevaluación y en las entrevistas individuales.

\section{- Sistema de calificación.}

Así, la evaluación final se manifiesta en una calificación, que se acompaña de observaciones y recomendaciones para que el alumno mejore. Los resultados de la evaluación se expresan en términos de insuficiente (1, 2, 3 o 4), suficiente (5), bien (6), notable (7 u 8) y sobresaliente (9 o 10).

Por lo tanto, al final del trimestre, la nota se obtiene de la media aritmética de las UUDD desarrolladas durante el mismo, la actitud y comportamiento, la participación, el "Classdojo", los juegos y trabajos grupales, el trabajo individual diario, el cuaderno y si traen ropa y calzado adecuados.

Todo esto se recoge en la ficha de calificación. En el caso de que no salga un número entero, se tiene en cuenta la progresión, ascendente o descendente de cada alumno. Esta es la única calificación que se dará a los alumnos a lo largo del trimestre. Pero de acuerdo con Pérez Pueyo \& López Pastor (2010), se va a otorgar porcentajes a los instrumentos de evaluación y no a conceptos, procedimientos y actitudes, ya que estos están englobados en los ítems que forman dichos instrumentos (ver tabla 1). 
Tabla 1.

Criterios e instrumentos de calificación.

\begin{tabular}{|c|c|c|}
\hline Criterios de calificación & Técnicas e instrumentos & Valoración \\
\hline $\begin{array}{c}\text { Participación y trabajo individual } \\
\text { diario en clase (Valoración de los } \\
\text { contenidos y del desarrollo de las } \\
\text { competencias adquiridas). }\end{array}$ & - $\quad$ Cuaderno o diario del maestro & $25 \%$ \\
\hline Objetivos alcanzados & Ficha de heteroevaluación & $25 \%$ \\
\hline $\begin{array}{c}\text { Punto extras (positivos o } \\
\text { negativos) }\end{array}$ & - $\quad$ Aplicación en el móvil (Classdojo) & $10 \%$ \\
\hline Cuaderno & $\begin{array}{l}\text { *Imprescindible entregar el cuaderno } \\
\text { para hacer media. }\end{array}$ & $10 \%$ \\
\hline Autoevaluación y coevaluación & $\begin{array}{c}\text { - } \quad \text { Fichas de autoevaluación y } \\
\text { coevaluación }\end{array}$ & --- \\
\hline $\begin{array}{c}\text { Actitud y comportamiento } \\
\text { Escucha, Esfuerzo, Colaboración, } \\
\text { Respeto, ayuda de material. }\end{array}$ & - $\quad$ Cuaderno o diario del maestro & $20 \%$ \\
\hline Ropa y calzado adecuado & $\begin{array}{c}\text { - Cuaderno o diario del maestro } \\
\text { *3 días sin traer el chándal es un } 0 \text { en } \\
\text { este apartado. }\end{array}$ & $10 \%$ \\
\hline
\end{tabular}

Tabla 2

Coherencia interna entre los elementos curriculares. Alineación curricular.

\begin{tabular}{|c|c|c|c|c|}
\hline $\begin{array}{l}\text { Finalidades ylo } \\
\text { Competencias }\end{array}$ & $\begin{array}{l}\text { Actividades } \\
\text { Aprendizaje }\end{array}$ & Evaluación formativa & $\begin{array}{l}\text { Instrumentos } \\
\text { calificación }\end{array}$ & $\begin{array}{l}\text { Criterios } \\
\text { calificación }\end{array}$ \\
\hline $\begin{array}{l}\text { - Conocer su cuerpo y la AF } \\
\text { como medio de disfrute y } \\
\text { relación con los demás. } \\
\text { - Utilizar las HMB para } \\
\text { adaptar el movimiento a los } \\
\text { diferentes juegos y } \\
\text { actividades. } \\
\text { - Realizar actividades que } \\
\text { ayuden a tomar conciencia }\end{array}$ & $\begin{array}{l}\text { Juegos motores } \\
\text { y actividades } \\
\text { físicas } \\
\text { organizadas }\end{array}$ & $\begin{array}{l}\text { Fichas autoevaluación } \\
\text { (se realizan al final de } \\
\text { la UD y posteriormente } \\
\text { se devuelve en clase } \\
\text { con comentarios } \\
\text { realizados por mí). }\end{array}$ & $\begin{array}{c}\text {-Ficha de } \\
\text { observación grupal. } \\
\text { - Diario del maestro. } \\
\text {-Ficha de } \\
\text { calificación. } \\
\text {-Ficha de } \\
\text { autoevaluación del } \\
\text { maestro y de las } \\
\text { UUDD. }\end{array}$ & $\begin{array}{c}\text { Participación } \\
\text { Implicación } \\
\text { Cooperación } \\
\text { Aspectos motores } \\
\text { (25\% de la nota } \\
\text { final) }\end{array}$ \\
\hline $\begin{array}{l}\text { de la importancia del } \\
\text { ejercicio físico, la higiene, la } \\
\text { seguridad y prevención de } \\
\text { riesgos, los primeros } \\
\text { auxilios, la alimentación y } \\
\text { los hábitos posturales. }\end{array}$ & $\begin{array}{l}\text { Actividad grupal } \\
\text { con coevalua- } \\
\text { ción }\end{array}$ & $\begin{array}{c}\text { Fichas de } \\
\text { coevaluación (en } \\
\text { proyectos grupales y } \\
\text { se comentan en la } \\
\text { misma sesión en gran } \\
\text { grupo). } \\
\end{array}$ & -No se califica. & \\
\hline $\begin{array}{l}\text { - Conocer y utilizar nociones } \\
\text { básicas asociadas al } \\
\text { espacio, al tiempo y a la } \\
\text { percepción y estructuración } \\
\text { espacio-temporal en el } \\
\text { centro. }\end{array}$ & $\begin{array}{c}\text { Asambleas } \\
\text { finales grupales }\end{array}$ & $\begin{array}{c}\text { Reflexiones orales en } \\
\text { gran grupo. Se refleja } \\
\text { a través de la } \\
\text { asignación de } \\
\text { pokemons. }\end{array}$ & $\begin{array}{l}\text {-Tabla } 5 \text { de } \\
\text { asignación de puntos } \\
\text { colgada en clase. }\end{array}$ & $\begin{array}{l}\text { Actitud en clase. } \\
\text { Respeto normas, } \\
\text { compañeros y } \\
\text { material. } \\
\text { Participación. }\end{array}$ \\
\hline $\begin{array}{l}\text { - Tomar conciencia de las } \\
\text { posibilidades de expresión y } \\
\text { comunicación del cuerpo a } \\
\text { través de la expresión } \\
\text { corporal. } \\
\text { - Iniciarse en la práctica de }\end{array}$ & Classdojo & $\begin{array}{c}\text { Reflexiones } \\
\text { individuales diarias. }\end{array}$ & $\begin{array}{l}\text { - Aplicación digital } \\
\text { educativa. } \\
\text {-Ficha de } \\
\text { calificación. }\end{array}$ & $\begin{array}{c}\text { Traer los } \\
\text { materiales que se } \\
\text { piden. } \\
\text { Autonomía. } \\
\text { (10\% de la nota } \\
\text { final) }\end{array}$ \\
\hline $\begin{array}{l}\text { diferentes juegos y deportes } \\
\text { a través de los juegos } \\
\text { modificados, así como de } \\
\text { algunos } \\
\text { alternativos. }\end{array}$ & Cuaderno & $\begin{array}{l}\text { Realizan en él una } \\
\text { actividad cada UD. } \\
\text { Este lo corrijo al final } \\
\text { de cada trimestre y se } \\
\text { lo devuelvo con las } \\
\text { correcciones } \\
\text { realizadas. }\end{array}$ & $\begin{array}{l}\text {-Ficha de } \\
\text { calificación. }\end{array}$ & $\begin{array}{l}\text { Capacidad de } \\
\text { reflexión sobre el } \\
\text { aprendizaje. } \\
\\
\text { (10\% de la nota } \\
\text { final) }\end{array}$ \\
\hline
\end{tabular}




\section{RESULTADOS}

Finalmente, se muestran los resultados académicos obtenidos en cada trimestre (Ver tabla 3), pudiendo realizar una comparación entre ellos, que detallo a continuación.

Tabla 3.

Comparación de resultados obtenidos.

\begin{tabular}{ccccccc}
\hline $\begin{array}{c}\text { Resultados } \\
\text { globales }\end{array}$ & \multicolumn{2}{c}{$\mathbf{1}^{\text {er }}$ trimestre } & \multicolumn{2}{c}{$\mathbf{2}^{\mathbf{0}}$ trimestre } & \multicolumn{2}{c}{$\mathbf{3}^{\text {er }}$ trimestre } \\
\hline Calificación & $\%$ & $\begin{array}{c}\mathbf{N}^{\mathbf{0}} \\
\text { alumnos/as }\end{array}$ & $\mathbf{\%}$ & $\begin{array}{c}\mathbf{N}^{\mathbf{0}} \\
\text { alumnos/as }\end{array}$ & $\mathbf{\%}$ & $\begin{array}{c}\mathbf{N}^{\mathbf{0}} \\
\text { alumnos/as }\end{array}$ \\
\hline Sobresaliente & 28,9 & 11 & 23,7 & 9 & 34,21 & 13 \\
\hline Notable & 52,6 & 20 & 57,9 & 22 & 39,47 & 15 \\
\hline Bien & 15,8 & 6 & 18,4 & 7 & 18,4 & 7 \\
\hline Suficiente & 0,03 & 1 & 0 & 0 & 0 & 0 \\
\hline Insuficiente & 0 & 0 & 0 & 0 & 7,89 & $3^{*}$ \\
\hline Totales & $\mathbf{1 0 0 \%}$ & $\mathbf{3 8}$ & $\mathbf{1 0 0 \%}$ & $\mathbf{3 8}$ & $\mathbf{1 0 0 \%}$ & $\mathbf{3 8}$ \\
\hline
\end{tabular}

En general, los resultados del primer trimestre son bastante satisfactorios. Los criterios que se utilizan para la calificación permiten que todos los alumnos, sean más o menos ágiles en EF, obtengan un buen resultado, ya que se valora mucho el esfuerzo, participación y trabajo diario.

En relación al segundo trimestre, las notas son muy similares, pero el número de sobresalientes ha disminuido y en los notables muchos 8 han bajado a 7 . Esto se debe en gran medida al cuaderno, ya que muchos alumnos lo han realizado incorrectamente y no se han esforzado lo suficiente en la reflexión de los contenidos. Por eso, se decide bajar las notas, intentando así, dar un toque de atención a los alumnos y que vuelvan a esforzarse y trabajar en el tercer trimestre.

En cuanto al tercer trimestre, los resultados por lo general han mejorado, ya que los alumnos han entendido lo que se pide en la asignatura y se han esforzado por conseguirlo. Aquellos que nos han mejorado, ha sido por falta de interés propia. Resaltar que hay 3 alumnos que han suspendido este trimestre por no entregarme el cuaderno, ya que es un requisito imprescindible y después de insistir, no han mostrado interés ninguno. En la nota final, ningún alumno suspende, ya que la media de los tres trimestres supera el 5.

\section{- Interpretación de dichos resultados.}

Como se ha podido comprobar, los resultados son, por lo general, muy satisfactorios. Esto se debe en gran medida a la evaluación compartida y formativa, ya que los alumnos conocen desde el primer momento los ítems y criterios a valorar y tener en cuenta, lo que les hace tenerlos presentes en las diferentes sesiones. Además, las constantes reflexiones les hacen ser conscientes de su aprendizaje en todo momento.

Por lo que debido a esta forma de evaluar, la mayoría de los alumnos saben perfectamente cómo se han portado, si han participado o no, como ha sido su actitud, etc., por lo que conocen casi con exactitud la nota que van a tener al final del trimestre, obteniendo resultados muy similares a los míos cuando comparo su autoevaluación y autocalificación con mis observaciones y calificaciones obtenidas. Así, vemos que en un $90 \%$ de los casos su nota coincide con la mía. Además, conseguimos así, que por un momento se olviden de eso que tanto les preocupa... LA NOTA. 
Destacar que, aunque se produce un descenso de las calificaciones en el $2^{\circ}$ trimestre, bajando las notas de algunos alumnos, estos se esfuerzan más, haciendo que sus calificaciones mejoren en el $3^{\text {er }}$ trimestre.

Pero además, podemos observar, que este método de evaluación provoca una serie de ventajas, como son:

- Interés mostrado por el alumnado en la evaluación y en el aprendizaje de la asignatura.

- Entienden que la Educación Física no es una asignatura donde se viene a jugar, sino que detrás de los juegos y actividades realizadas hay un aprendizaje significativo, que pueden extrapolar a su día a día.

- Consiente un conocimiento más individualizado del alumnado, sobre todo cuando tenemos alumnos nuevos a los que aún no conocemos.

- Permite a los alumnos a darse cuenta de su comportamiento y actitud en clase, de su trabajo diario, de su participación, de la importancia de los contenidos y las actividades etc., es decir, les ayuda a mejorar su capacidad de reflexión y su espíritu crítico.

- La autoevaluación grupal a través de la asignación de puntos me permite que los alumnos estén motivados en las clases de EF aún más, así como que su comportamiento sea mucho mejor. Además, los alumnos dialogan entre ellos cuando hacen cosas que no deben para intentar conseguir el pokemon más alto.

- Hace que los alumnos quieran trabajar con los compañeros que mejor trabajan y no solo con sus amigos. Al principio, cuando mandaba crear grupos libres de trabajo siempre se ponían con sus amigos, lo que les perjudicaba en gran medida, puesto que no dejaban de hablar, pero con el paso de las sesiones, se han dado cuenta que es más beneficioso ponerse con otras personas con las que trabajan mejor.

- Por lo general, se muestran de acuerdo con la calificación obtenida al final del trimestre, ya que son partícipes de la misma en todo momento.

$Y$ también, podemos encontrarnos con inconvenientes, que nos ayudan a buscar soluciones para mejorar nuestro proceso de evaluación compartida y formativa y continuar aprendiendo y mejorando, como podemos ver en la siguiente tabla (figura 3).

\begin{tabular}{|c|c|}
\hline Principales inconvenientes encontrados & Posibles soluciones de mejora \\
\hline Lleva bastante tiempo de elaboración. & Rehusar fichas ya elaboradas o simplificarlas. \\
\hline $\begin{array}{l}\text { En las fichas de autoevaluación, algunos } \\
\text { alumnos se ponen menos nota de la que se } \\
\text { merecen en determinados aspectos. }\end{array}$ & $\begin{array}{l}\text { Hablar con ellos, y decirles que sean realistas } \\
\text { y observen bien los comentarios que yo les } \\
\text { hago. }\end{array}$ \\
\hline $\begin{array}{l}\text { Formular ítems positivos y negativos dentro } \\
\text { de la misma ficha de auto o coevaluación, } \\
\text { ya que muchas veces no leen bien y lo } \\
\text { marcan mal inconscientemente. }\end{array}$ & Formular todos los ítems en positivo. \\
\hline $\begin{array}{l}\text { Hacer } 3 \text { niveles dentro de las rúbricas o } \\
\text { fichas de autoevaluación, ya que los niños } \\
\text { me pedían ponerse en un punto intermedio. }\end{array}$ & $\begin{array}{l}\text { Ampliarlo a más niveles. A mí me ha } \\
\text { funcionado ponerlo en } 5 .\end{array}$ \\
\hline Algunos alumnos no hacen el cuaderno. & $\begin{array}{l}\text { Suspenderles para darles un toque de } \\
\text { atención, porque si les aprobamos van a } \\
\text { seguir haciendo lo que quieren. } \\
\text { Introducir el cuaderno desde el primer } \\
\text { trimestre para que tengan mayor tiempo de } \\
\text { asimilación del mismo. }\end{array}$ \\
\hline
\end{tabular}

Figura 3. Principales inconvenientes encontrados y posibles soluciones para cada uno de ellos. 
Falta de costumbre del proceso de evaluación compartida y formativa.

Falta de tiempo en la aplicación de algunos instrumentos.

Falta de participación de algunos alumnos en las asambleas finales.
Irles introduciendo poco a poco en el mismo, y aunque al principio se quejen, no hacerles caso hasta que se acostumbren.

Adelantar la realización de algunos instrumentos, sobre todo en el $3^{\text {er }}$ trimestre, ya que en junio se pierden muchas clases.

Tener un mayor control y registro de los que participan y obligar a participar a aquellos que no lo hacen.

Figura 3. Principales inconvenientes encontrados y posibles soluciones para cada uno de ellos (continuación).

\section{CONCLUSIONES}

En conclusión, podemos observar, que aplicar este tipo de evaluación supone, ya no solo mejores resultados, si no que los niños sean conscientes de su propio aprendizaje, ya que participan como protagonistas en su propio proceso de evaluación. Aunque, al no estar acostumbrados a ello, cuando se aplican nuevos instrumentos, su calificación baja, pero al final se produce una mejora notable. Al principio es algo nuevo para ellos, pero según se avanza en el tiempo son más participes del proceso evaluativo y eso es lo que más me gusta de la evaluación formativa y compartida, que los propios alumnos son participes de su evaluación y conscientes de lo que se pide.

Además, este sistema supone una motivación extra para los alumnos, ya que se les hace partícipe de la evaluación y se intenta quitar importancia a la nota, valorando así el proceso y no tanto el resultado. Debido a esto, vemos como los niños cada vez que terminamos la unidad, me piden la ficha de autoevaluación y una vez que ha sido revisada por mí, me la vuelven a pedir, para ver si su opinión coincide con la mía.

Por otro lado, y cuando los alumnos van asimilando este sistema de evaluación formativa, trabajan mucho más rápido, pues sus ideas son más claras y los instrumentos utilizados ya les son conocidos. Por lo que lo que al principio parecía un inconveniente de tiempo, ahora es una gran ventaja, que se ha ido obteniendo con el tiempo.

Aunque este sistema de evaluación formativa y compartida es costoso, puesto que exige un mayor trabajo por parte del profesor que lo utiliza, merece la pena, ya que el esfuerzo empleado se recompensa con el aprendizaje del alumnado, ayudando al cumplimiento de los objetivos propuestos.

\section{REFERENCIAS}

López-Pastor V. M., \& Pérez-Pueyo, A. (coords.) (2017). Evaluación formativa y compartida en Educación: experiencias de éxito en todas las etapas educativas. León: Universidad de León.

Pérez-Pueyo, A., \& López-Pastor V. M. (2017). Introducción a la creación y uso de escalas descriptivas y rúbricas. En V. M. López-Pastor \& A. Pérez-Pueyo (coords.) Evaluación formativa y compartida en Educación: experiencias de éxito en todas las etapas educativas, (pp. 92-115). León: Universidad de León.

Ruíz Nebrera, J. J. (2008). La evaluación de la Educación Física en la educación primaria: mecanismos e instrumentos. Revista EF y Deportes, 121. Recuperado de: https://bit.ly/30UjaMy 